ordinary transparent media $U$ is so small compared with $u$ that it may be neglected, and puts it zero everywhere.

The results of the various theories differ in the form they give for the dispersion formula. Lommel's theory has been shown by Voigt to be untenable. The theories of Helmholtz, Thomson, and Sellmeier lead, when $b$ is small, to the same result, and give

which Ketteler's gives

$$
\mu^{2}=\mathrm{I}+\frac{\beta^{2} \tau^{2}}{\rho}\left\{\mathrm{I}+\Sigma \frac{q \tau^{2}}{\tau^{2}-\kappa^{2}}\right\},
$$

$$
\mu^{2}=\mathrm{I}+\frac{\beta^{2}}{\rho}+\Sigma_{\tau^{2}-\kappa^{2}}{ }^{2}
$$

$\tau$ is the period of the ether vibration, $\kappa$ of the matter vibration, and $q, D, \&$ c, , are functions of the constants.

Voigt's formula, since he does not consider the matter motion, is different and not so general.

With regard to these formulæ, I am not aware that Helmholtz's has been tested by comparison with experiment. Ketteler's has, and agrees excellently over a long range of values of $\tau$.

Double refraction is generally explained by supposing $\beta^{2}$ to be a function of the direction; but, as Sir W. Thomson has pointed out, this involves for Helmholtz's theory-he did not, however, apply his formulæ to crystals-dispersion with double refraction. For Ketteler's theory this is not the case. $\mu$ can be a function of the direction independently of $\tau$.

The mechanism which would make the action between the matter and ether in each element of volume a function of the acceleration is perhaps not so easy to conceive as that supposed by Helmholtz and Thomson; but still Ketteler's theory seems to overcome some of the difficulties inherent in the latter.

Either of these theories can be shown to lead to Fresnel's wave-surface, provided we do not consider it necessary that the vibrations should lie in the wave-front. The vibration, as indeed Ketteler and Boussinesq have pointed out, will be normal to the ray. In all other respects Fresnel's construction will hold.

Ketteler and Voigt have tried, without much success, to apply their theories to reflection and refraction.

Thomson, in that most valuable appendix to his Baltimore lectures, has given a complete theory. This can be readily adapted to Ketteler's theory, and the results in many points agree in a striking manner with experiments both for transparent and opaque bodies. The occurrence of a real negative value for $\mu^{2}$ is explained by the supposition that the period of the incident light is higher than the highest possible mode of vibration for the matter-molecules in the medium.

The last section deals with Maxwell's electro-magnetic theory of light.

Electro-magnetic disturbance travels in air. with a velocity equal to that of light; and in a double refracting medium obeys Fresnel's laws. The difficulty lies in giving a physical explanation of light motions, and of accounting for the mechanical structure of the ether required by the theory. No complete theory of dispersion has yet been given. The work of Willard Gibbs does not explain why there is no dispersion in a vacuum. The objection made to Cauchy's theory holds good. It is probable that some theory such as is developed in the third section may be successfully applied to the electro-magnetic disturbance.

The theory has the great advantage of connecting naturally with the theory of light the important electro and magnetooptical discoveries of Faraday, Kerr, Kundt, and Quincke, and to the development of this much is due to Prof. Fitzgerald. The theory of reflection and refraction as at present developed is only approximate.

\section{ELECTROL YSIS}

PROF. LODGE opened the discussion at the Aberdeen meeting of the British Association on Electrolysis by reading a paper, the notes of which have already appeared in NATURE.

Sir W. Thomson referred, in his remarks on Prof. Lodge's paper, to a matter of importance in electro-plating-viz. the selection which takes place in the electrolysis of solutions containing several salts, as, for instance, in the electrolysis of copper sulphate containing ferrous sulphate, which, when decomposed by a strong current gives a deposit containing impurities, whereas a slower decomposition yields a very pure deposit. Sir W. Thomson spoke also of the necessity for the careful investigation of those cases in which the formation of deposits between the electrodes had been observed, and it would be important to know whether deposits could be formed in the line of conduction without a nucleus at all. Such matters are of importance to physiology, indicating a possible danger in the passing of long continued currents through the human body.

Prof. Schuster explained the views propounded by Von Helmholtz in his recent papers on this subject. Helmholtz explains the phenomena of electrolysis by assuming a different attraction of different chemical elements for electricity. If this be admitted, most of the difficulties connected with the phenomena of contact electricity disappear. In electrolysis the element (say hydrogen) charged with positive electricity travels to the negative electrode and forms a coating over it. Any electromotive force, however small, is sufficient to produce this effect, as no work is done. The hydrogen does not appear as free hydrogen, however. It is only liberated when the electromotive force is sufficient to produce a transfer of the positive electricity from the hydrogen molecule to the electrode. When the dissociated elements appear in a neutral state an interchange of the electricities of the elements must have occurred before dissociation. In this way we may explain the conversion of stannic in stannous chloride, which was mentioned by Prof. Armstrong in his address. Prof. Schuster did not think that Prof. Lodge had laid sufficient stress on the fact that in very dilute solutions an ion has the same rate of transference, no matter with what element it was combined. This fact affords strong evidence in favour of the above views, from which it follows as a necessary result. Prof. Schuster also explained his own views of the discharge of electricity in gases. He believes that the phenomena present some analogy to those exhibited in electrolysis of liquids. The phenomena exhibited at the negative pole are, he thinks, due to dissociation of the compound molecule. They do not appear in the case of monatomic mercury vapour. Experiments which he hopes to conclude in the next few months will decide whether or not the law of the constancy of molecular charge holds.

The next contribution to the discussion was a paper by Dr. C. R. Alder Wright, containing an account of the nature of his investigations, conducted with the view of measuring Chemical Affinity in terms of E.M.F.

On the Sensitiveness to Light of Selenium and Sulphur Cells, by Shelford Bidwell, M.A., LL.B. - The author suggests that the operation of annealing in the making of selenium cells increases the sensitiveness to light by promoting the combination of the selenium with the metal of the electrodes, forming a selenide which completely surrounds the electrodes, and is, perhaps, diffused throughout the selenium when in a liquid condition; further, that the apparently improved conductivity of the selenium, together with the electrolytic phenomena which it exhibits, are to be accounted for by the existence ot this selenide. This view finds considerable support in the fact that cells, constructed with sulphur, replacing the selenium and containing a proportion of silver sulphide, are all more or less sensitive to light, and exhibit properties of annealed selenium. The author also read a paper On the Generation of a Voltaic Current by a Sulphur Cell with a Solid Electrolyte, a short account of which has already appeared in NATURE (vol. xxxii. p. 345).

\section{MOLECULAR WEIGHTS}

THE discussion on the Molecular Weights of Liquids and solids was opened in Section B of the British Association by the reading of a paper by Prof. A. W. Reinold, F.R.S., the subject of which was the Size of Molecules. In this paper an account was given of the different lines of argument by which Sir W. Thomson has been led to form an estimate of the size of molecules. The estimate is based upon four lines of argument-the first, from the refractive dispersion of light; the second, from the phenomena of contact electricity; the third, from liquid films; and the fourth, from the kinetic theory of gases. All four agree in showing that in liquids and transparent solids the mean distance between the centre of contiguous molecules is something between $\mathbf{I} /$ roth and $\mathbf{I} / 200$ th of a millionth of a millimetre. Recently Exner (Monatschrift fïr Chemie, vi. 244-278) has proposed another method for estimating the diameter of gaseous molecules, the results obtained by this method being slightly smaller than those deduced from the above. The author gave an account of his experiments on soap-films, conducted conjointly with Prof. Rucker (NATURE, vol. xxxii. p. 210), the results of which are 